\title{
Image Case:Ocular Toxocariasis in 14 Years Old Male.
}

\author{
Tarik I Zaher ${ }^{1}$, Ahmed S Mohammed ${ }^{1}$, Mohammed Abozaid ${ }^{2}$, \\ Ashraf Mahrous Eid Mohammed ${ }^{3}$ \\ ${ }^{1}$ Tropical Medicine Department, Faculty of Medicine, Zagazig University, Egypt. \\ ${ }^{2}$ Endemic Medicine Departement, Administration of Health, Sharkia Governorate,Egypt.. \\ 3 Ophthalmology Department, Faculty of Medicine, Zagazig University, Egypt. \\ tareqzaher@gmail.com
}

Toxocariasis is one of zoonotic infections all over the world that is caused by Toxocara canis and Toxocara cati. Infection with Toxocara occurs by accidentlal ingestion of embryonated eggs or larvae which have been shed in the feces of infected animals. After ingestion of the eggs, release of infective larvae in the small intestine occurs and then these penetrate the intestine then to the circulation, and then to different organs[1]. Clinical presentation of toxocariasis in humans varies from asymptomatic infection to severe organ damage, which depends on the load of the parasite, the involved organs, and inflammatory response of the host. Particularly, depending on the involved organ, two clinical types can occur: systemic toxocariasis (which is known as visceral larva migrans) and ocular toxocariasis [2].

The majority of the ocular toxocariasis cases are unilateral. To reach definitive diagnosis of ocular toxocariasis, biopsy of infected tissue should be obtained for histological demonstration of the Toxocara larva which is difficult in clinical practice. So, we depend on the presence of typical ophthalmologic signs and the presence of serum antibody to the Toxocara larvae for diagnosis of ocular toxocariasis $[3,4]$.

A 14 years old male presented by progressive decrease in visual acuity in the left eye with no history of trauma or medications, with blood picture showed no esinophilia. Slit lamp examination was normal. Coloured fundus photography and fluorescein angiography of the left eye revealed evidence of fibrous band stretching from the optic disc to a peripheral retinal mass located in the far peripheral nasal retina which is highly suggestive of ocular toxocariasis ( figure 1).

Patient received medical treatment in the form of systemic corticosteroids $(1 \mathrm{mg} / \mathrm{kg})$ and diethyl carbamazine ( $6 \mathrm{mg} / \mathrm{kg}$ per day) for one month then reexamination was done and coloured fundus photography of the eye revealed marked improvement of the traction bands on the macula with improvement of visual acuity ( figure 2).

Ethical consideration: Consent was obtained from the case. All the information gathered from the patient was handled confidentially, and it was used only for research purpose.

Funding: None.

Conflict of interest: There is no conflict of interest.

\section{REFERENCES:}

1.Rubinsky-Elefant G, Hirata CE, Yamamoto JH, Ferreira MU. Human toxocariasis: diagnosis, worldwide seroprevalences and clinical expression of the systemic and ocular forms. Ann Trop Med Parasitol 2010;104:3-23.

2. Stewart JM, Cubillan LD, Cunningham ET Jr. Prevalence, clinical features, and causes of vision loss among patients with ocular toxocariasis. Retina 2005;25:1005-13.

3.Gillespie SH, Dinning WJ, Voller A, Crowcroft NS. The spectrum of ocular toxocariasis. Eye (Lond) 1993;7(Pt 3):415-8.

4.Yokoi K, Goto H, Sakai J, Usui M. Clinical features of ocular toxocariasis in Japan. Ocul Immunol Inflamm 2003;11:269-7. 


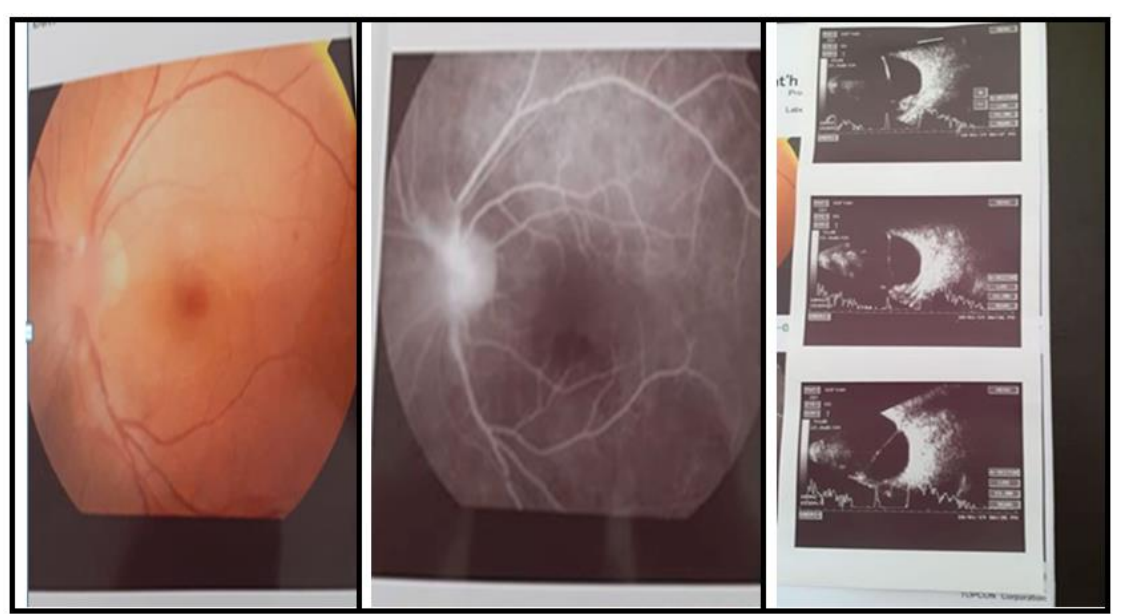

Figure (1): Coloured fundus photography and fluorescein angiography of the eye showing fibrous band stretching from the optic disc to a peripheral retinal mass located in the far peripheral nasal retina which is highly suggestive of ocular toxocariasis.

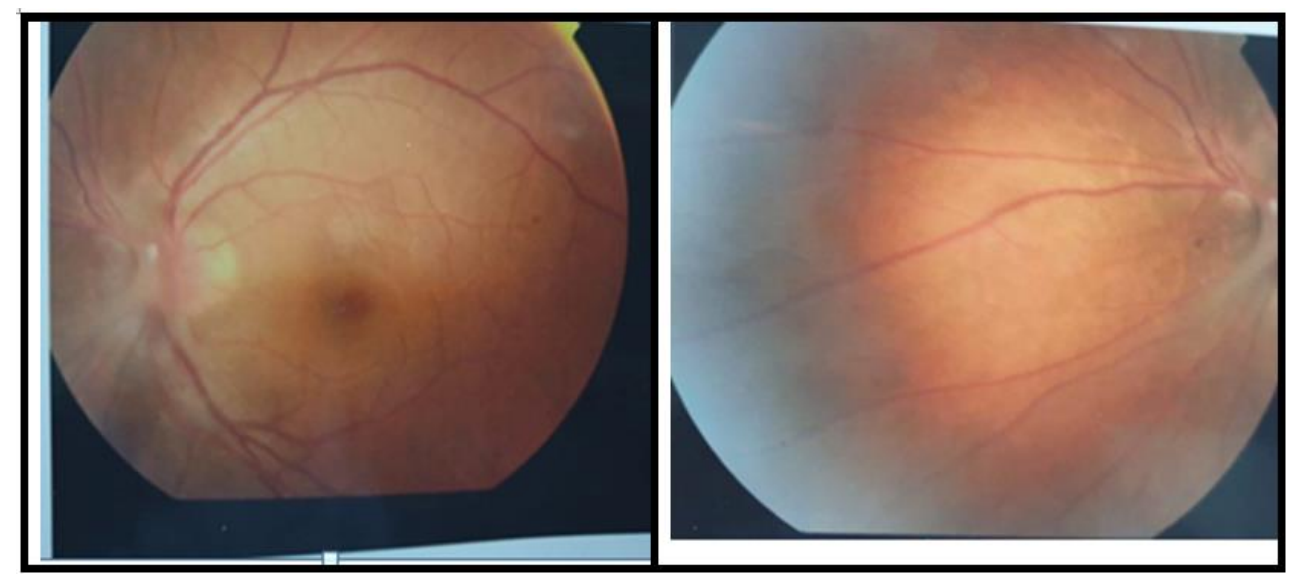

Figure (2): Coloured fundus photography of the eye revealed improvement of the traction of the macula after one month of treatment. 